\title{
LES CENTRIFUGEUSES ACUTRONIC
}

\author{
J. NICOLAS-FONT
}

ACUTRONIC - BP 64 - 8 rue deS Dames - 78340 LES CLAYES SOUS BOIS

\section{ABSTRACT}

The paper describes the main activities that ACUTRONIC FRANCE Society develops in the design of important centrifuges for testing. These activities interest mainly two areas: geotechnical engineering and biomechanical engineering. A list of the operational geotechnical centrifuges over the world is given.

\section{GENERALITES}

II existe entre autres dans le catalogue ACUTRONIC FRANCE des grandes centrifugeuses d'essais, deux familles:

- les centrifugeuses géotechniques

- les centrifugeuses de bio-mécanique

A chacune de ces familles de machines correspondent des caractéristiques de fonctionnement précises et distinctes, correspondant bien entendus aux besoins différents exprimés par les utilisateurs.

\section{SPECIFICATIONS}

Afin de bien décrire les différences qu'il existe entre les deux familles de centrifugeuses, le tableau ci-après fait le parallèle sur les points essentiels de leur fonctionnement. 


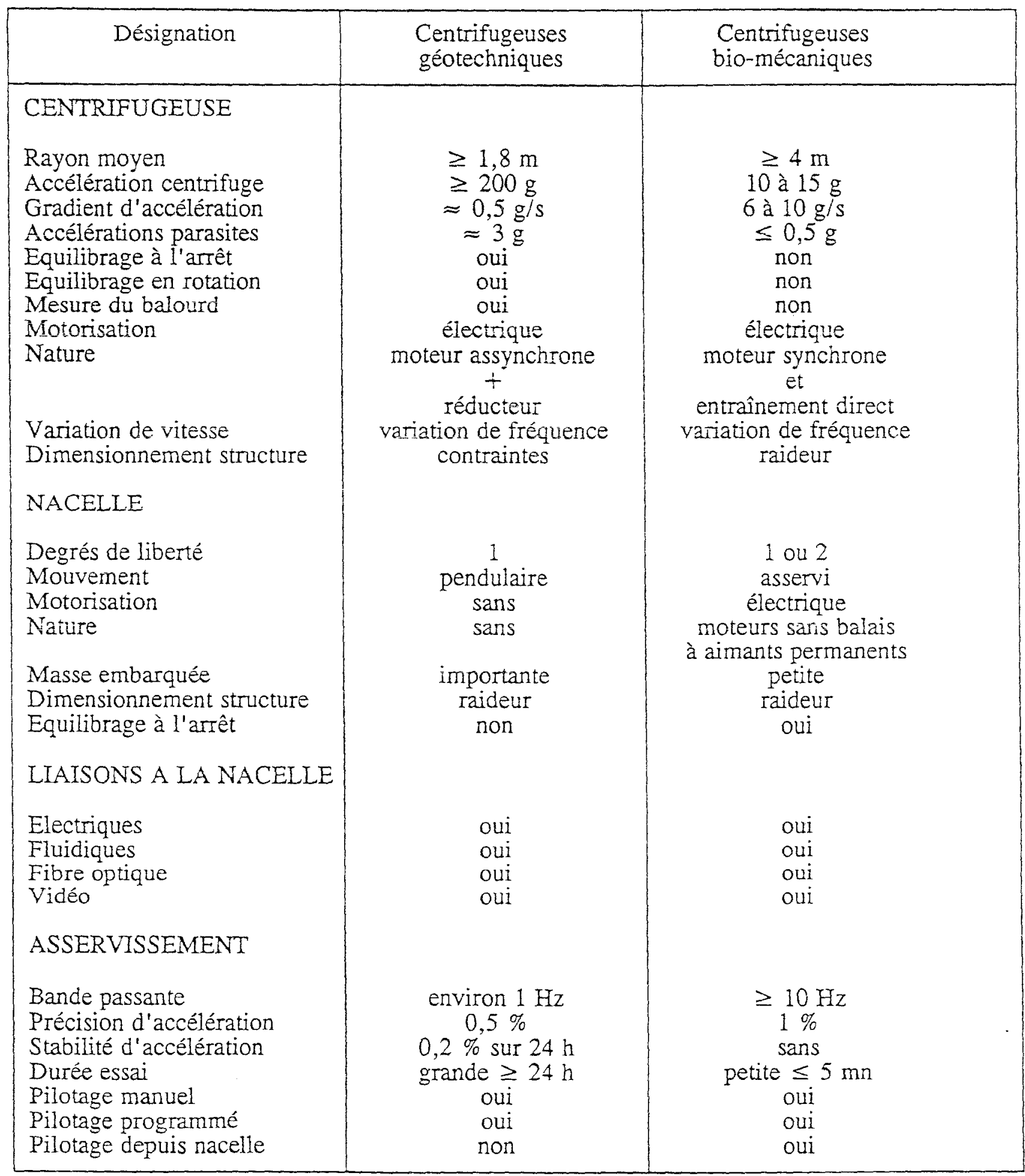




\section{CENTRIFUGEUSES GEOTECHNIQUES}

Il est inutile de répéter la description de ces centrifugeuses pour lesquelles it existe une bibliographie importante. En ce qui concerne les centrifugeuses ACUTRONIC il est possible de se référer à J. NICOLAS-FONT (1988, 1990 eł 1991).

Il est important de remarquer que les possibilités d'essais offertes par la simulation en centrifugeuse sont de plus en plus appréciées par les ingénieurs en mécanique de sols. Cet enjouement traduit sans aucun doute les bons résultats que permet d'obtenir cette technique et se trouve être en corrélation avec la croissance du nombre des centrifugeuses existant dans le monde

Sans vouloir être exhaustifs, les tableaux des pages suivantes donnent la liste des centrifugeuses existantes ou en projet actuellement. Cette liste est classée par pays. L'attention du lecteur est attirée sur le fait que charge maximale et accélération maximale ne vont pas de paire. 


\begin{tabular}{|c|c|c|c|c|c|c|}
\hline PAYS & ORGANISHE & $\begin{array}{l}\text { ANNEE DE } \\
\text { MISE EN } \\
\text { OEUVRE }\end{array}$ & $\begin{array}{c}\text { RAYON A LA } \\
\text { PLATEFORME } \\
\text { (m) }\end{array}$ & $\begin{array}{l}\text { CHARGE } \\
\text { MAXIMALE } \\
\text { (kg) }\end{array}$ & $\begin{array}{c}\text { ACCELERATION } \\
\text { MAXIMALE } \\
\text { (g) }\end{array}$ & CONSTRUCTEUR \\
\hline \multirow[t]{2}{*}{ All emagne } & Un. Bochum & 1987 & 4.125 & 2000 & 250 & Krupp \\
\hline & Un.8ochem & 1992 & 1.8 & 500 & 200 & Acutronic $(661-1)$ \\
\hline Australie & UWA & 1989 & 1.8 & 500 & 200 & Acutronic $(861-1)$ \\
\hline \multirow[t]{3}{*}{ Canada } & Queen's Un* & & & 1 & 20000 & \\
\hline & Queen's un & & 2.68 & & 270 & \\
\hline & C.Core & 1993 & 5.5 & 2000 & 200 & Acutronic $(680-2)$ \\
\hline \multirow[t]{7}{*}{ Chine } & UN. Hehai & 1974 & 2.4 & 100 & 250 & \\
\hline & NRHI & 1982 & 2.9 & 100 & 200 & \\
\hline & NRHI & 1990 & 5 & 2000 & 200 & \\
\hline & CHIDI* & 1985 & 10.8 & 3000 & 110 & \\
\hline & YRSRI & 1985 & 3 & 1000 & 300 & \\
\hline & $S R I$ & 9987 & 1.55 & 100 & 200 & \\
\hline & IWHR & 1990 & 4 & 1500 & 300 & \\
\hline \multirow[t]{2}{*}{ Danemark } & DEA & 1973 & 0.65 & 25 & 1000 & \\
\hline & $D E A$ & 1975 & 2.3 & 1250 & 80 & \\
\hline \multirow[t]{2}{*}{ France } & CEA-CESTA* & 1964 & 10.5 & 2000 & 100 & Larécoère \\
\hline & LCPC & 1986 & 5.5 & 2000 & 200 & Acutronic $(680)$ \\
\hline \multicolumn{7}{|l|}{ Grande- } \\
\hline \multirow[t]{7}{*}{ Bretagne } & UMIST & 1969 & 1.5 & 750 & 150 & \\
\hline & simon & 1971 & & 5500 & 200 & \\
\hline & Un.Cambridge & 1974 & 4.3 & 900 & 170 & UN. Cambridge \\
\hline & Un.Liverpool & 1974 & 1.15 & 200 & 200 & Triotech \\
\hline & City Un. & 1988 & 1.8 & 500 & 200 & Acutronic (661) \\
\hline & Un. Manchester & & 3.2 & 5500 & 140 & \\
\hline & Luton & & & 225 & 80 & \\
\hline Italie & ISMES & 1988 & 2 & 400 & 600 & \\
\hline \multirow[t]{11}{*}{ Japon } & Un.osaka & 1965 & 2.56 & 200 & 200 & \\
\hline & Un. Chuo & 1987 & 3.05 & 550 & 180 & \\
\hline & Un.Chuo & & 1.18 & 150 & 270 & \\
\hline & Kajima & 1990 & 3 & 1000 & 200 & Acutronic $(665-1)$ \\
\hline & Nikken Sekkei & 1992 & 3 & 1000 & 200 & Acutronic $(665-1)$ \\
\hline & PHR I & & 3.8 & 2700 & 115 & \\
\hline & TIT & 1970 & 1.18 & & 300 & \\
\hline & TIT & & 1.26 & 250 & 150 & \\
\hline & PWR I & & 1.25 & 130 & 360 & \\
\hline & Toyo & & 2.20 & 300 & 250 & \\
\hline & Takenaka & 1993 & 7.0 & 5000 & 200 & Acutronic $(685-1)$ \\
\hline Pays-Bas & Un.Delft & 1989 & 6 & 5500 & 300 & Schenck \\
\hline Portugal & LNEC & 1992 & 1.8 & 500 & 200 & Acutronic (661-1) \\
\hline
\end{tabular}




\begin{tabular}{|c|c|c|c|c|c|c|}
\hline PAYS & ORGANISME & $\begin{array}{l}\text { ANNEE DE } \\
\text { MISE EN } \\
\text { OEUVRE }\end{array}$ & $\begin{array}{c}\text { RAYON A LA } \\
\text { PLATEFORME } \\
(m)\end{array}$ & $\begin{array}{l}\text { CHARGE } \\
\text { MAXIMALE } \\
(\mathrm{kg})\end{array}$ & $\begin{array}{l}\text { ACCELERATION } \\
\text { MAXIMALE } \\
(g)\end{array}$ & CONSTRUCTEUR \\
\hline $\begin{array}{l}\text { URSS } \\
\text { USA }\end{array}$ & $\begin{array}{c}\text { MIIT } \\
\text { NIIS } \\
\text { AZNIISM } \\
\text { TSNIIS } \\
\text { Un.Kiev } \\
\text { MSM } \\
\text { Sandia* } \\
\text { Sandia } \\
\text { Un.Boulder } \\
\text { Un.Boulder } \\
\text { Un.Davis } \\
\text { Un.Davis } \\
\text { Un.Californie } \\
\text { Un.Californie } \\
\text { RPI } \\
\text { Boeing* } \\
\text { Caltech } \\
\text { Un.Princeton } \\
\text { Un.Maryland } \\
\text { MIT } \\
\text { Cl } \\
\text { Un.Floride } \\
\text { Un.Floride } \\
\text { Tyndall AFB* } \\
\text { Upsalla* } \\
\text { Un.Columbia* } \\
\text { Us Corp.Eng. }\end{array}$ & $\begin{array}{l}1956 \\
1968 \\
1988 \\
1989 \\
1989 \\
1989\end{array}$ & $\begin{array}{l}2.5 \\
2.5 \\
5.5 \\
2.5 \\
2.5 \\
0.9 \\
7.5 \\
2.13 \\
5.49 \\
1.36 \\
9.1 \\
1 \\
\\
3 \\
1.3 \\
1.83 \\
0.5 \\
1\end{array}$ & $\begin{array}{c}2 \times 170 \\
2 \times 170 \\
2500 \\
\\
2200 \\
\\
\\
3500 \\
250 \\
2000 \\
136 \\
3600 \\
\\
4000 \\
100 \\
1000 \\
200 \\
40 \\
100 \\
75 \\
75 \\
60 \\
50 \\
150 \\
10 \\
1 \\
6000\end{array}$ & $\begin{array}{l}320 \\
320 \\
500 \\
95 \\
300 \\
\\
\\
200 \\
150 \\
200 \\
100 \\
300 \\
\\
100 \\
100 \\
200 \\
600 \\
175 \\
100 \\
200 \\
200 \\
120 \\
100 \\
100 \\
4000 \\
2500 \\
350\end{array}$ & $\begin{array}{c}\text { Genisco } \\
\text { Schacvitz } \\
\text { Acutronic (665-1) } \\
\text { Boeing } \\
\text { Genisco } \\
\text { Acutronic }(684-1)\end{array}$ \\
\hline
\end{tabular}

* Installation non spécifique au Génie Civil 


\section{CENTRIFUGEUSES BIO-MECANIQUES}

comme leur nom générique l'indique ces centrifugeuses sont destinées à l'étude du comportement mécanique de modèles biologiques sous l'effet de fortes accélérations. Le fonctionnement de ces centrifugeuses simule essentiellement les accélérations produites par les avions de combat modernes afin de permettre létude des phénomènes entrainant la perte rapide de connaissance subies par les pilotes.

Ces pertes de connaissance se produisent brusquement. sans signes avantcoureurs, et entrainent dans la majorité des cas la perte du pilote et de l'avion: entre 1980 et 1986 les Etats Unis (US Air Force) ont perdu au moins 30 appareils, en général avec mort des pilotes (P. QUANDIEU). La cause principale de cette perte de connaissance est la diminution du flux sanguin irrigant le cerveau d"un sujet soumis à une forte accélération dont la direction est paralèle di la colonne vertébrale.

Les objectifs des esscis sur centrifugeuses sont par conséquent:

- lentrainement des pilotes

- la recherche médicale

- la recherche sur les appareillages permettant daugmenter le domaine de tolérance.

\section{Iechnologies adoptées par ACUTRONIC}

Afin dobtenir les performances dynamiques voulues pour ces centrifugeuses (gradient d'accélération jusqu'à $10 \mathrm{~g} / \mathrm{s}$ ). ACUTRONIC a adopté les technologies suivantes

- Réalisation de la centrifugeuse en matéraiux composites afin d'augmenter le rapport raideur / masse, ce qui permet l'optimisation de la puissance nécessaire à la motorisation qui est malgré tout de l'ordre de $1000 \mathrm{~kW}$

- Réalisation dune motorisation électrique par moteur triphasé synchrone basse vitesse ( $50 \mathrm{tr} / \mathrm{mn}$ max) et entrainement direct du bras de la centrifugeuse car les cycles de fonctionnement sont courts mais très "hachés" (variation environ toutes les secondes du sens du couple moteur maximal transmis). 
- Réalisation en bout de bras d'une nacelle à deux dégrés de liberté asservis en position afin d'orienter parfaitement la colonne vertébrale du sujet suivant la direction de l'accélération résultante centrifuge + tangentielle + pesanteur).

- Réalisation de moteurs triphasés à aimants permanents sans balais pour la motorisation des axes nacelles.

- Mise en rotation de la centrifugeuse de façon a obtenir des variations linéaires d'accélération résultante.

\section{Réalisations ACUTRONIC}

A l'heure actuelle ACUTRONIC a réalisé une centrifugeuse bio-mécanique de $4 \mathrm{~m}$ de rayon avec une nacelle à un degré de liberté et permettant l'emport d'une charge utile de $40 \mathrm{~kg}$. Laccélération maximale de cette centrifugeuse est de $15 \mathrm{~g}$ avec un gradient de $6 \mathrm{~g} / \mathrm{s}$. Cette centrifugeuse est installée au CERMA (Service Médical de l'Armée de l'Air Française), dans l'enceinte du Centre d'Essais en Vol de Brétigny.

Une autre centrifugeuse plus importante est en cours de réalisation pour une mise en route en 1993. Elle a om de rayon avec une nacelle à deux degrés de liberté et permet lemport d'une charge utile de $200 \mathrm{~kg}$. L'accélération maximale de cette centrifugeuse est de $10 \mathrm{~g}$ avec un gradient de $6 \mathrm{~g} / \mathrm{s}$. Elle est destinèe à l'entrainement des pilotes de chasse de l'Armée de l'Air Française

\section{Perspectives}

Bien qu'il existe déjà par le monde bon nombre de centrifugeuses biomécaniques à faible dynamique (environ $\mathrm{lg} / \mathrm{s}$ ) répondant aux performances des avions de chasse développés après la deuxième guerre mondiale, cellesci sont inadaptées aux exigences des avions actueis et encore moins des avions futurs.

II parait donc évident que l'Armée de l'Air dans son ensemble souhaite, à plus ou moins longue échéance, acquérir une centrifugeuse moderne ayant les capacités dynamiques voulues. L'action d'ACUTRONIC sinscrit dans ce cadre et, bien que la concurrence dans ce domaine soit plus sévère que dans le domaine géotechnique. nous pensons construire une vingtaine de centrifugeuses sur une période de dix ans. 


\section{BIBLIOGRAPHIE}

(1) J. NICOLAS-FONT : Design of geotechnical centrifuge, Centrifuge 88, Corté 1988 Balkema, Rotterdam.

(2) J. NICOLAS-FONT: Sur la conception des centrifugeuses géotechniques, Premières Journées Inter-universitaires 1990. Université du Havre, Université de Nantes

(3) J. NICOLAS-FONT: Design, construction and operation of 100 g-ton centrifuge at RPl, Centrifuge 91. Hom-Yim ko 1991 A.A. Balkema. Rotterdam, Brookfield.

(4) P. quandieu, D. GafFie, PH. liebaert, M. briane, j.C. Sarron, A. GUILLAUME, D. TRAN et J. PH. HAYMAN: G-LOC, Gz et hypoxie cérébrale $G z / s$ ef hypertension intracranienne. AGARD conference proceedings 516 distribué par ONERA 92220 Chatillon/Bagneux. 